\title{
Multiscale Modelling for Cerebrospinal Fluid Dynamics: Multicompartmental Poroelacticity and the Role of AQP4
}

\author{
Dean Chou ${ }^{1,2}$, John C. Vardakis ${ }^{1,2}$, Yiannis Ventikos ${ }^{3}$ \\ ${ }^{1}$ Institute of Biomedical Engineering, University of Oxford, Oxford, UK \\ ${ }^{2}$ Department of Engineering Science, University of Oxford, Oxford, UK \\ ${ }^{3}$ Department of Mechanical Engineering, University College London, London, UK \\ Email: y.ventikos@ucl.ac.uk
}

Received November 2013

\begin{abstract}
Cerebrospinal fluid (CSF) is recognized to play an important role in the brain environment and central nervous system (CNS). At the microscopic level, glial cells and water channel proteins (WCPs), also known as aquaporins (AQPs), are believed to be central in regulating CSF. Furthermore, such elements are postulated to associate with numerous cerebral and neurological pathologies. The novelty of the present research is the attempt to investigate such pathophysiological phenomena via a multi scale physical model incorporating mechanisms across all scales, including the AQP effects. The proposed physical multiscale model can explore the relationship between CSF and glial cells via the incorporation of AQPs (as microscopic channels) and elaborate on the macroscopic manifestations of this interplay. This study aims to make a tangible contribution to the understanding of cerebral or neurological pathologies via virtual physiological human (VPH) in silico.
\end{abstract}

\section{Keywords}

\section{Aquaporin; Hydrocephalus; Cerebrospinal Fluid; Computational Fluid Dynamics}

\section{Introduction}

Neurocognitive conditions are getting more common, especially if the aging population is taken into account. Brain science focused on modern lifestyle diseases is thus receiving increasing attention. Various brain diseases are related to imbalance in water regulation [1]. Not making any attempt to be exhaustive, we can mention tumours that may lead to obstructions and problems in absorption of CSF; strokes and head impact injuries leading to oedemas, malformations of the cerebral ventricles and passages resulting in hydrocephalus or Chiari disease, and elevated intracranial pressure that may lead to ischemic dementia [2]. Although the relationship between water transport and the function of each component of the central nervous system (CNS) still appears as, more or less, an enigma because of the complexity of the human brain, it plays an important role in its physiology and pathology. For instance, the regulation of the water balance in the different fluid compartments of the brain via 
the activity of water channel proteins (WCPs) is a critical issue which has not been fully explored.

In recent years many neuroscience research groups have made attempts to address topics concerning WCPs. After the discovery of the first AQP (AQP1) in red blood cell membranes, other WCPs have been discovered in various parts of the human body, including the brain and the nervous system (NS), where they are believed to play a role in physiology and pathology [3] [4]. AQP effects are not well understood, especially when the different interplaying scales of the cerebral environment are considered.

We propose extending the Multiple-Network Poroelastic Theory (presented previously by the group) with an AQP mechanism (MPET-AQP). Further to this, we propose to embed the new model within a finite-volume computational fluid dynamics (CFD) framework, for the purpose of studying, in detail, the transport of water within a patient-specific cerebral environment reconstructed from T2 weighted MRI data. The advantage of using the MPET-AQP formulation is that it allows the investigation of fluid transport phenomena regarding CSF, brain parenchyma and cerebral blood across all scale, in a physically consistent and comprehensive manner.

\section{Methodology}

The one-dimensional consolidation of clay soils was described by Karl von Terzaghi who coined the term poroelastic theory [5]. Biot introduced a three-dimensional generalization of the poroelastic theory in 1941 [6] to describe the settlement of soil under a load where the mechanism of consolidation is similar to the process of squeezing fluid from an elastic porous medium (like a sponge). Since then, this theory has had wide applications in Mechanical Engineering, e.g. two phase flows [7] and in Civil Engineering (e.g. Geotechnical Engineering [8]). This framework has also been applied on biological soft tissues, for the last 10 - 15 years [9]-[13]. At the macroscopic level, it is rational that we base our analysis on the MPET model in order to illustrate the behavior of CSF in the parenchyma within the cerebral environment (shown in Figure 1).

\subsection{MPET-AQP Model}

This research proposes a novel application of Multiple-Network Poroelastic Theory (MPET) to investigate water transport in the brain. This study adopts an extension of Biot'sporoelastic theory [5] which was initially used to describe the relationship between the pressure of a fluid permeating a solid matrix and its constituent displacement (consolidation). The system equations for the MPET model are can be cast as:

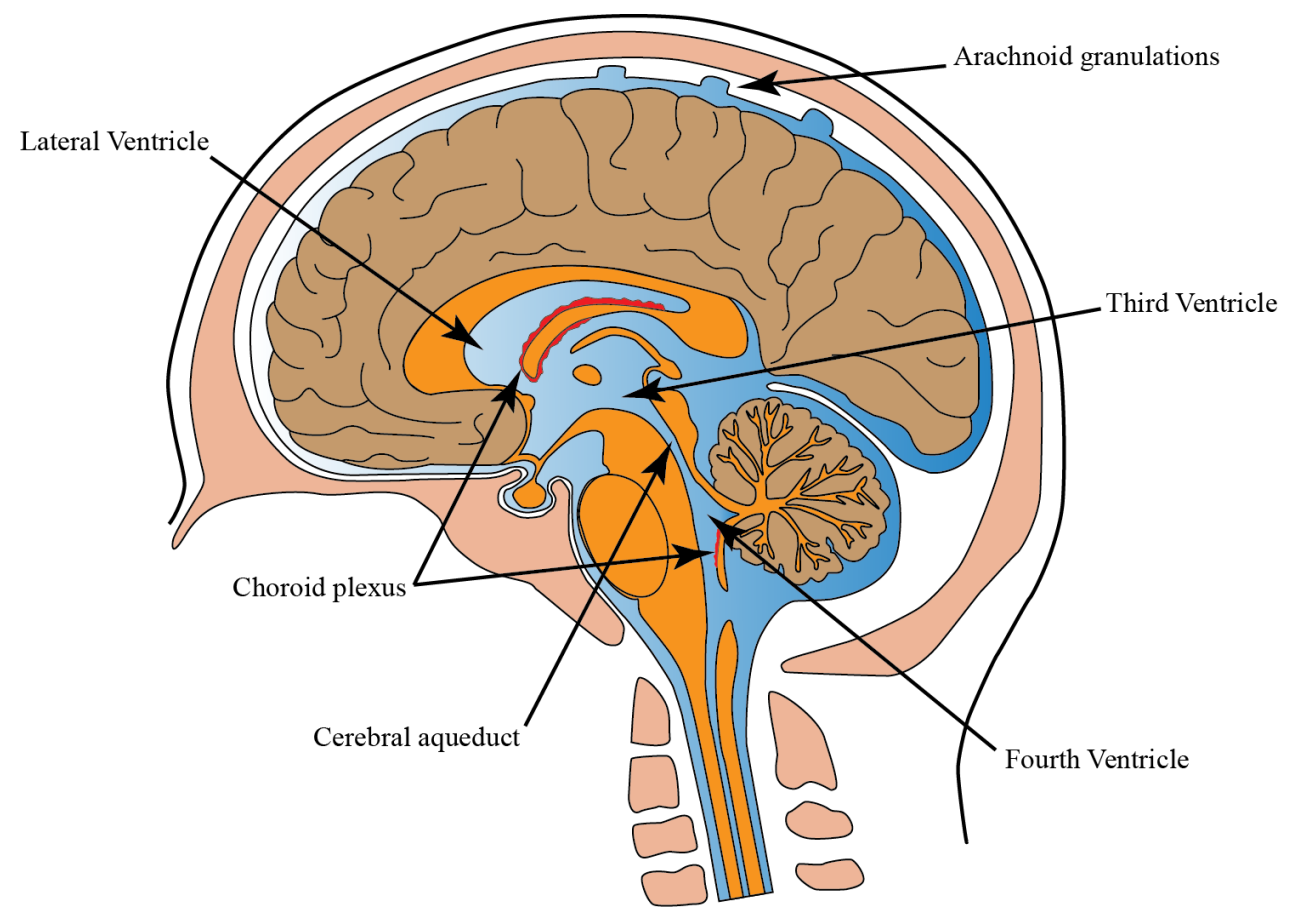

Figure 1. The ventricular system of the human brain. 


$$
\nabla \cdot \sigma^{M}+\rho_{f}\left(f^{b}-\frac{\partial^{2} u}{\partial t^{2}}\right)-\sum_{\beta=1}^{A} \alpha^{\beta} \nabla p^{\beta}=0
$$

where del, $\nabla$, is defined in terms of partial derivative operators; $\sigma^{M}$, is the effective stress vector; $\rho_{f}$, is the density of the fluid phase; $f^{b}$, is the body force vector, and $\boldsymbol{u}$, is the displacement vector; $\alpha^{\beta}$ is the Biot parameter of the fluid network.

$$
\frac{1}{Q^{\beta}} \frac{\partial p^{\beta}}{\partial t}+\alpha^{\beta} \frac{\partial(\nabla \cdot u)}{\partial t}-\sum_{b=1, b \neq \beta}^{A} \dot{S}_{b \rightarrow \beta}+\nabla \cdot\left[\kappa^{\beta} \rho_{f}^{\beta}\left(f^{b}-\frac{\partial^{2} u}{\partial t^{2}}\right)-\kappa^{\beta} \nabla p^{\beta}\right]=0
$$

where $Q^{\beta}$, is the amalgamated compressibility of both fluid and solid phases; $\dot{S}_{b \rightarrow \beta}$, is the rate of fluid exchange between any two networks; $\kappa^{\beta}$, represents the anisotropic permeability coefficient.

MPEt allows a novel extension of traditional poroelastic models to include detailed transfer mechanisms between the cerebral blood and extracellular fluid/CSF (and generally, any other compartment deemed necessary). The four fluid networks concept is shown in Figure 2. This biological system is based on two assumptions regarding fluid networks concerning wall thickness and leakiness of blood vessels in the system. The first assumption is that the characterization of the network keeps directional flow transport. The second assumption is that the MPET framework could be considered as a quasi-steady system, hence, $\partial^{2} / \partial t^{2} \rightarrow 0$ and $\partial / \partial t \rightarrow 0-$ this assumption is specific to the clinical problems we are interested in (like for example HCP) that involve very slow development (order of days or weeks) and can thus be viewed as quasi-steady.

Meanwhile, we have tried to establish a simple, heuristic, AQP model, implementing it and embedding it within the MPET framework and assessing the impact of AQP expression by varying $\dot{S}_{c e}$. We attempted to use the following equation to demonstrate the selective permeability of the AQP behaviour in the cerebral aqueduct:

$$
\kappa_{A Q P}=\kappa_{e}\left[1-\left(\frac{p_{e}-p_{\text {ref }}}{p_{\text {ref }}}\right)\right] A_{f}
$$

where $\kappa_{e}$ is the base permeability between extracellular and intracellular space in the CSF compartment, $p_{e}$ is the CSF pressure, $p_{r e f}$ is a reference pressure, and $A_{f}$ is an amplification factor (heuristic at the present stage).

The basic idea here is that the above-defined permeability depends on the porosity of this compartment. The porosity (ratio of free space over total volume or equivalently difference of total minus occupied volume over total volume) depends on the volume of the astrocytes. Therefore astrocytes may swell or shrink via water transport through their AQPs - that are speculatively "gated" by pressure [14]. Therefore, the purpose of introducing such a relationship is to examine the potential influence of the pressure-controlled permeability of this compartment. Since the arbitrary amplification factor can assume relatively large values and the dependence of permeability on porosity is a complex affair, it has been shown that, quantitatively, it can involve very substantial changes [15].

\subsection{Boundary Conditions}

We discuss the boundary conditions that close the system.

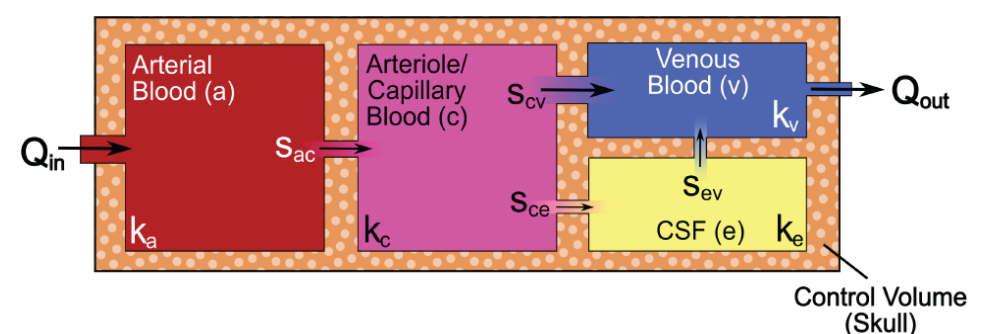

Figure 2. The schematic illustration of the transfer restrictions set in the MPET model [16]. 


\subsubsection{Skull}

The shell responsible for outlining the skull lies at $r=r_{M}$, and at the same location, the displacement is considered as naught. Hence, we have the boundary condition of a rigid skull (as in adults), and it could be representedas:

$$
u\left(r_{M}, t\right)=0
$$

The blood pressures in the arterial and venous compartments are given by:

$$
p^{a}\left(r_{M}, t\right)=\tilde{p}_{b p}
$$

and,

$$
p^{v}\left(r_{M}, t\right)=p_{b p}
$$

Moreover, the above boundary conditions have the restriction for the inlet and outlet flow of the capillary network, $c$, at the skull and the accumulation of CSF of the venous network, $v$. The following equations assume that the CSF absorption induces a pressure rise and zero deformation of a purely rigid skull.

$$
\frac{\partial p^{c}\left(r_{M}, t\right)}{\partial t}=0
$$

A pressure rise depends on the resistance to absorption, $R$, and the rate of absorption, $Q_{C S F}^{e}$, is presented as:

$$
p^{e}\left(r_{M}, t\right)=p^{v}\left(r_{M}, t\right)+\mu^{e} R Q_{C S F}^{e}
$$

where $\mu^{e}$ is the dynamic viscosity of the CSF, $R$ is the outflow resistance due to the arachnoid granulations, and $Q_{C S F}^{e}$ is the efflux of CSF at the region of the skull.

\subsubsection{Ventricular Wall}

Since the stress is assumed continuous across the wall, we have the following boundary conditions at $r=r_{v}$,

$$
(1-v) \frac{\partial u\left(r_{v}, t\right)}{\partial r}+2 v \frac{u\left(r_{v}, t\right)}{r_{v}}-\sum_{\beta=a, e, c, v} \frac{\left(\alpha^{\beta}-1\right)(1-2 v)}{2 G} p^{\beta}\left(r_{v}, t\right)=0
$$

The reduced pressure gradient occurs in the capillary compartment due to the production of CSF from the blood. Hence, we have:

$$
-\kappa_{c \rightarrow v e n} \frac{\partial p^{c}\left(r_{v}, t\right)}{\partial r}=-Q_{C S F}
$$

where $\kappa_{c \rightarrow v e n}$ is the capillary network resistance to the flow from the capillary network, $c$.

Furthermore, no inlet and outlet flow between the arterial networks, $a$, and venous networks, $v$, exists (see Figure 2); then the equation is given as:

$$
\frac{\partial p^{a}\left(r_{v}, t\right)}{\partial r}=\frac{\partial p^{v}\left(r_{v}, t\right)}{\partial r}=0
$$

Finally, the conservation of bulk flow and momentum within the aqueductal system can be considered by utilizing Poiseuille's Law, in the first instance. It is assumed that the CSF is produced by the porous choroid plexuses, and it also accumulates within the ventricles and induces the cerebroventricular volume to increase. Hence, the following conditioncan be cast:

$$
Q_{C S F}=\frac{\pi d^{4}}{128 \mu^{e} L}\left[p^{e}\left(r_{v}, t\right)-p^{e}\left(r_{M}, t\right)\right]-\frac{4 \pi \kappa^{e}}{\mu}\left[r_{v}+u\left(r_{v}, t\right)\right] \frac{\partial p^{e}}{\partial r}+4 \pi\left[r_{v}+u\left(r_{v}, t\right)\right]^{2} \frac{\partial u}{\partial t}
$$

where $d$ is the diameter of the cerebral aqueduct, and $L$ is the length of the cerebral aqueduct (shown in Figure 3). 


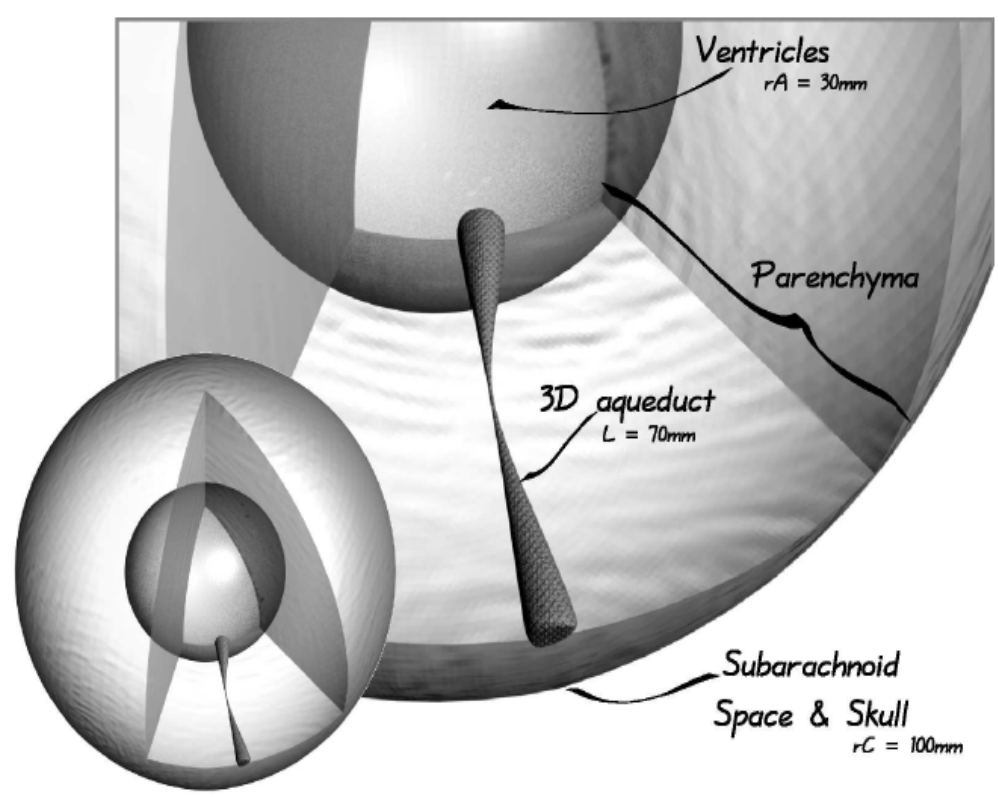

Figure 3. The concept of the 3D cerebral aqueduct in a spherically symmetricgeometry for the human brain [17].

\subsection{Numerical Implementation}

The governing MPET-AQP equations mentioned above are coupled with a solver that accounts for the full 3D unsteady momentum and continuity equations, as they apply to open, CSF-occupied, spaces (like ventricles and the cerebral aqueduct). These conservation equations are solved with a finite volume implicit second-order central finite difference scheme and with SIMPLEC (Semi-Implicit Method for Pressure-Linkage Equations Consistent) pressure-velocity coupling [18]. The coupling between the MPET-AQP solver and the flow solver is implemented via user-defined subroutines in CFD-ACE+.

After each time step of the MPET solver, the pressure at the skull and inside the ventricles is fed back to the user-defined subroutine as a pressure boundary condition. Following the solution of the aqueduct flow, the bulk flow is calculated in the user-defined subroutine and passed to the MPET-AQP solver for the next iteration.

\section{Results and Discussion}

Although the exact role of AQP4 in oedema (or in general) continues to be debated, it is known that the expression of AQP4 rapidly increases with oedema formation, thus there is evidence of correlation between water imbalances and AQP4 action [19]. We will present some initial results to demonstrate the potential importance of considering AQP effects.

We conduct two sets of numerical experiments: firstly, we test the heuristic AQP4 sensitization approach proposed, for an open (normal) cerebral aqueduct case. Secondly, we try the same approach to a case where the aqueduct is severely stenosed (i.e. a case where much of the produced CSF has to permeate and pass through the parenchyma - and thus experiences the permeability change effects that the CSF compartment undergoes under the influence of the AQP4 sensitization approach). The results we shall present will illustrate three interesting points:

a) Under the quasi-steady imposed conditions (the pulsatility is introduced via the boundary conditions only), the system of equations converges, in most cases, relatively quickly to steady values regarding pressure distributions and deformations in the parenchyma.

b) For the open cerebral aqueduct case, the AQP4 sensitization has a limited influence on the simulation results; however when the aqueduct is severely stenosed substantial differences between the AQP-insensitive and the AQP-sensitized models can be observed. Moreover, the specifics of this sensitization in terms of the permeability-pressure relationship also play a significant role, thus justifying exploring this theme further.

c) The coupled MPET-AQP model can provide valuable insight regarding the ventricular and aqueductal hy- 
drodynamics; in terms (for example) of flow patterns and pressure distribution in the aqueduct—a quantity of practical interest clinically.

Figure 4 shows the pressure distribution along a radius through the parenchyma and ventricles for the case of open cerebral aqueduct and for values of $\mathrm{A}=1$ and $\mathrm{A}=1000$ in the pressure-permeability AQP relationship. Additionally, it shows a similar setting but with a substantially augmented value of the amplification factor $(\mathrm{A}=$ 1000). This value does not influence the speed of convergence, at the same time it does not change the distribution of pressure either (the changes in shape and values for the converged pressure plots are negligible). It is even more interesting (but expected, from these pressure plots) to note that the corresponding ventricular displacement plots also show negligible differences when the permeability of the CSF compartment is adjusted and sensitized to pressure, with values tested spanning three orders of magnitude. This behavior however changes very substantially when the stenosed aqueduct case is taken into consideration. In this scenario, substantial flow is expected to "pass" through the porous parenchyma because the pressure drop generated at the aqueduct is substantial. Figure 5 demonstrates first that the rate at which pressure builds up to steady-state is dramatically delayed: it takes roughly 10 hours for the pressure profile to stabilize.

Moreover, as expected, the shape and values of the pressure profile are substantially different than those of the open cerebral aqueduct case (compare the dotted line in Figure 4 and Figure 5). Although the former discrepancy may be, partly, an artifact of the omission of the inertial terms in the basic poroelastic equations, the different shape and value (approximately $40 \%$ higher pressure) are converged effects.

Furthermore, Figure 6 demonstrates representative streamlines through the open cerebral aqueduct, severe cerebral aqueduct, as well as a cut through the cerebral aqueduct with the velocity magnitude distribution. The lower amplification factor induces a reduction of the peak of the velocity magnitude, as the alterations in the permeability would imply.

This exploratory investigation is concluded by examining the concomitant displacement fields that reflect of course the same message as the pressure conveys. Figure 7, presenting a selection only of the cases computed, compares the open and severe stenosed cerebral aqueduct cases for the same value of the amplification factor and shows a marked difference in the displacement field.

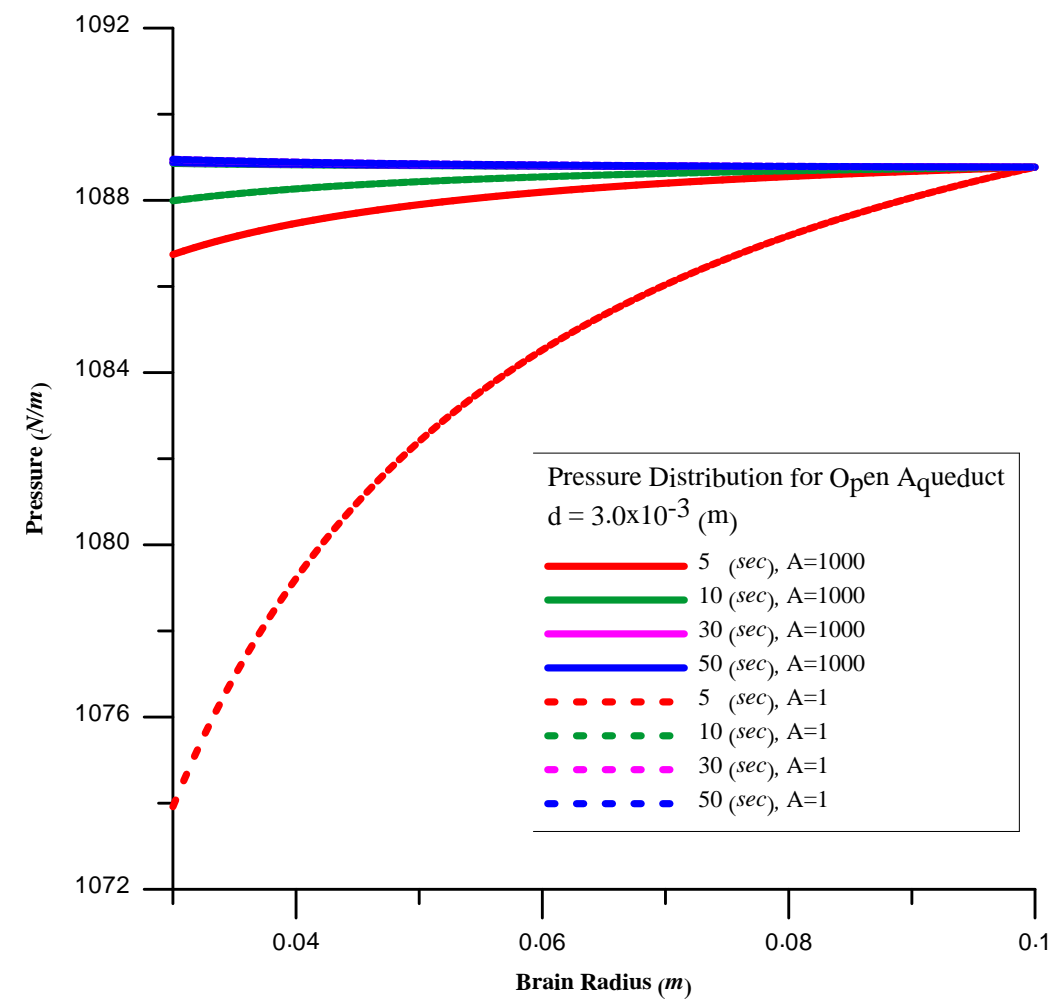

Figure 4. Convergence features and pressure distribution of the MPET-AQP model for open aqueduct case. 


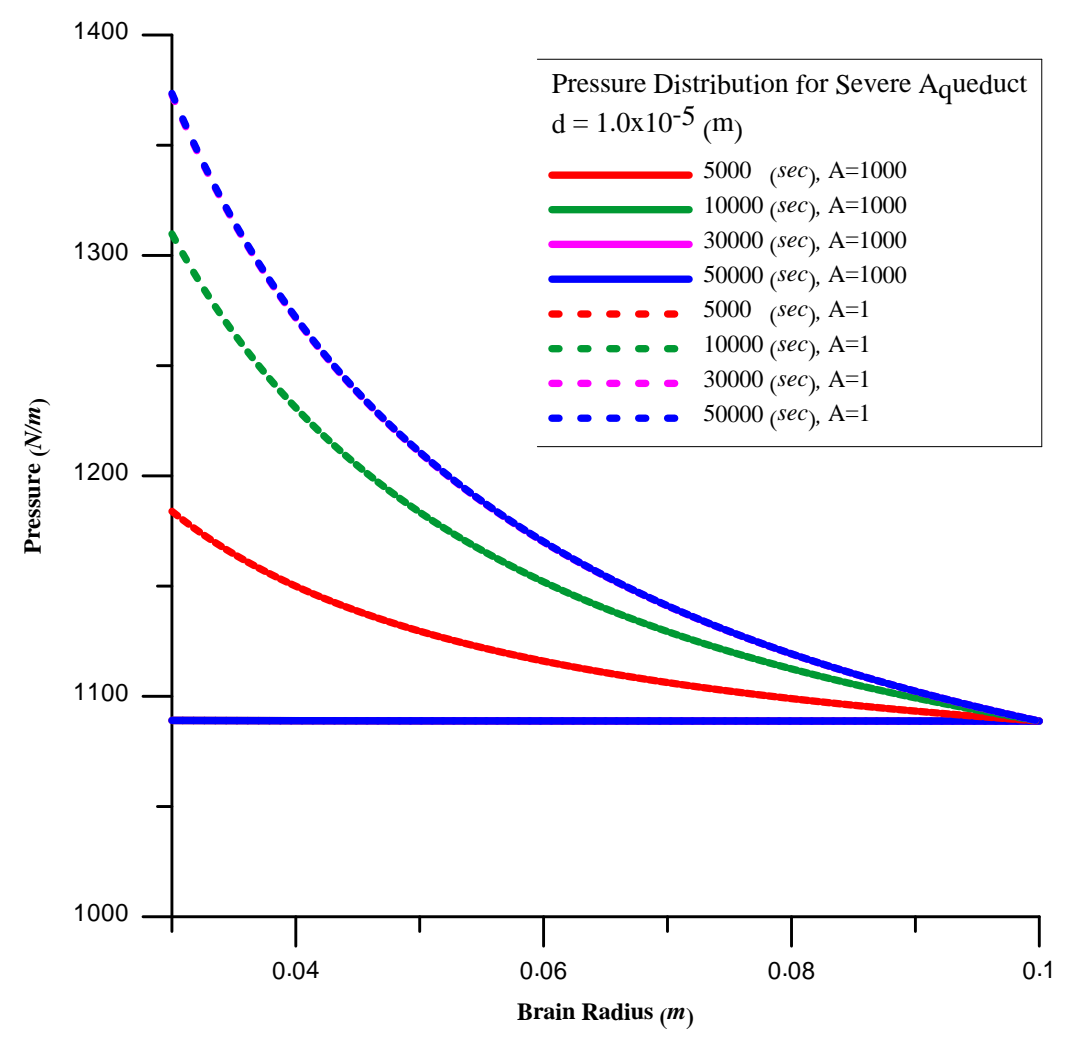

Figure 5. Transient behavior of pressure distribution-MPET-AQP model and severe aqueduct case.

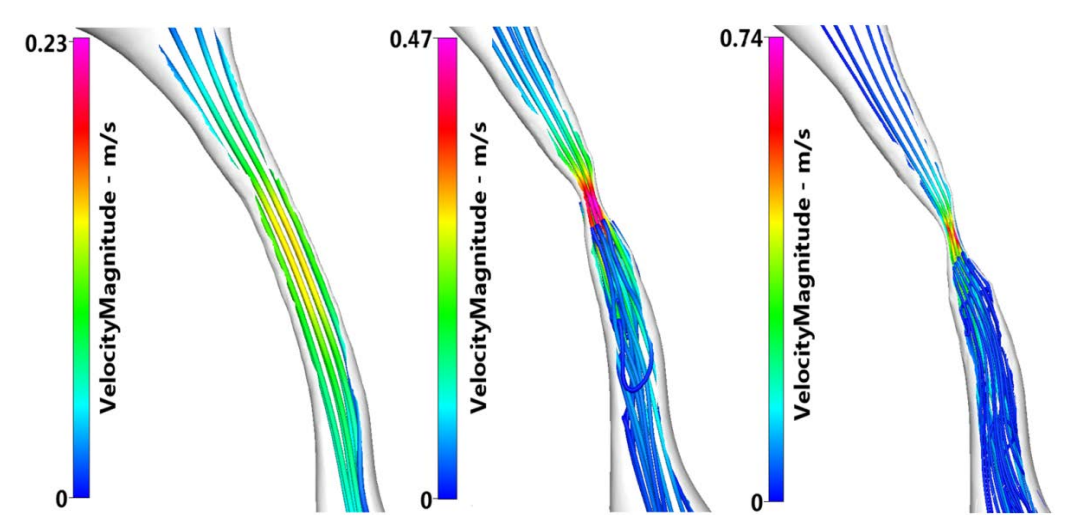

Figure 6. Open cerebral aqueduct in the left, severe cerebral aqueduct $(A=1)$ in the middle and severe cerebral aqueduct $(\mathrm{A}=10)$ in the right.

\section{Conclusion}

In this study, utilizing a novel implementation of the MPET-AQP model for integrative cerebral biomechanics, we have investigated the applicability and utility of such a multiscale framework for cerebral transport phenomena. This platform can assist in exploring unclear processes in the brain, such as the exact role of AQP4 in regulating water transport. We have demonstrated the coupling of the MPET-AQP model with a detailed patient specific 3D geometry, with unsteady ventriculoaqueductal hydrodynamics as well as a novel, albeit heuristic, relationship accounting for the cellular absorption of water through pressure-controlled AQP4. Early results are promising: the AQP-sensitized model shows that the AQPs may play an important role in obstructed aqueduct pathologies. Furthermore, a completely novel application of stent deployment has been proposed to replace current aqueductoplasty methods. 


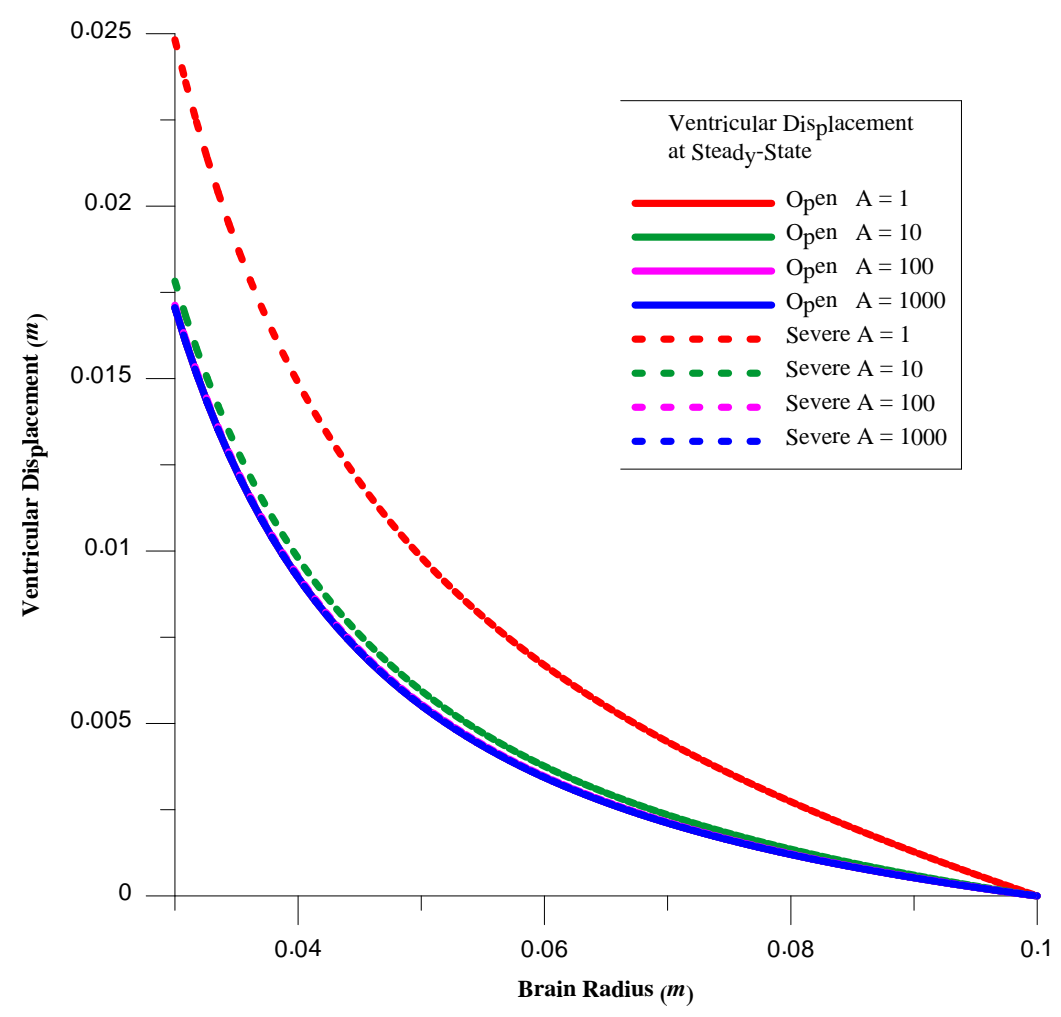

Figure 7. The influence of the amplification factor: comparison of severe vs open aqueduct.

\section{Acknowledgements}

We would like to thank the ESI-Group for allowing the use of the CFD-ACE+ multiphysics suite.

\section{References}

[1] Rosenberg, G.A. (1999) Ischemic Brain Edema. Progress in Cardiovascular Diseases, 42, 209-216. http://dx.doi.org/10.1016/S0033-0620(99)70003-4

[2] Rockwood, K., et al. (2007) Toward a Revision of Criteria for the Dementias. Alzheimer's \& Dementia, 3, $428-440$. http://dx.doi.org/10.1016/j.jalz.2007.07.014

[3] Hasegawa, H., et al. (1994) Molecular Cloning of a Mercurial-Insensitive Water Channel Expressed in Selected WaterTransporting Tissues. Journal of Biological Chemistry, 269, 5497-500.

[4] Jung, J.S., et al. (1994) Molecular Characterization of an Aquaporin cDNA from Brain: Candidate Osmoreceptor and Regulator of Water Balance. Proceedings of the National Academy of the United States of America, 91, 13052-13056. http://dx.doi.org/10.1073/pnas.91.26.13052

[5] Terzaghi, K. (1923) Die berechnung der durchlassigkeitzifer des tones aus dem verlauf der hydrodynamischen spannungserscheinungen, Mathematish-naturwissenschaftliche, Klasse. Akademie der Wissenschaften, Vienna, 125-138.

[6] Biot, M.A. (1941) General Theory of Three-Dimensional Consolidation. Journal of Applied Physics, 12, 155-164. http://dx.doi.org/10.1063/1.1712886

[7] Mendes, M.A., Murad, M.A. and Pereira, F. (2012) A New Computational Strategy for Solving Two-Phase Flow in Strongly Heterogeneous Poroelastic Media of Evolving Scales. International Journal for Numerical and Analytical Methods in Geomechanics, 36, 1683-1716. http://dx.doi.org/10.1002/nag.1067

[8] Carcione, J.M., Morency, C. and Santos, J.E. (2010) Computational Poroelasticity—A Review. Geophysics, 75, A229A243.

[9] Pena, A., Bolton, M.D. and Pickard, J.D. (1998) Cellular Poroelasticity: A Theoretical Model for Soft Tissue Mechanics. http://www-civ.eng.cam.ac.uk/geotech_new/people/bolton/mdb_pub/76_poromechanics98_475_480.PDF

[10] Stokes, I.A., et al. (2010) Limitation of Finite Element Analysis of Poroelastic Behavior of Biological Tissues Under- 
going Rapid Loading. Annals of Biomedical Engineering, 38, 1780-1788. http://dx.doi.org/10.1007/s10439-010-9938-0

[11] Wang, X. and Hong, W. (2012) A Visco-Poroelastic Theory for Polymeric Gels. Proceedings of the Royal Society A: Mathematical, Physical and Engineering Science.

[12] Polzer, S., et al. (2012) Impact of Poroelasticity of Intraluminal Thrombus on Wall Stress of Abdominal Aortic Aneurysms. BioMedical Engineering OnLine, 11, 62. http://dx.doi.org/10.1186/1475-925X-11-62

[13] Nia, H.T., et al. (2011) Poroelasticity of Cartilage at the Nanoscale. Biophysical Journal, 101, 2304-2313. http://dx.doi.org/10.1016/j.bpj.2011.09.011

[14] Wan, X.C., Steudle, E. and Hartung, W. (2004) Gating of Water Channels (Aquaporins) in Cortical Cells of Young Corn Roots by Mechanical Stimuli (pressure pulses): Effects of ABA and of $\mathrm{HgCl}$. Journal of Experimental Botany, 55, 411-422. http://dx.doi.org/10.1093/jxb/erh051

[15] Kimelberg, H.K. (2004) Water Homeostasis in the Brain: Basic Concepts. Neuroscience, 129, 851-860. http://dx.doi.org/10.1016/j.neuroscience.2004.07.033

[16] Tully, B. and Ventikos, Y. (2011) Cerebral Water Transport Using Multiple-Network Poroelastic Theory: Application to Normal Pressure Hydrocephalus. Journal of Fluid Mechanics, 667, 188-215. http://dx.doi.org/10.1017/S0022112010004428

[17] Tully, B. and Ventikos, Y. (2009) Coupling Poroelasticity and CFD for Cerebrospinal Fluid Hydrodynamics. IEEE Transactions on Biomedical Engineering, 56, 1644-1651. http://dx.doi.org/10.1109/TBME.2009.2016427

[18] Vandoormaal, J.P. and Raithby, G.D. (1984) Enhancements of the Simple Method for Predicting Incompressible FluidFlows. Numerical Heat Transfer, 7, 147-163.

[19] Badaut, J., Brunet, J.F. and Regli, L. (2007) Aquawporins in the Brain: From Aqueduct to "Multi-Duct”. Metabolic Brain Disease, 22, 251-263. http://dx.doi.org/10.1007/s11011-007-9057-2 